\title{
Prognostic significance of folate metabolism polymorphisms for lung cancer
}

\author{
A Matakidou ${ }^{1,3}, \mathbf{R}$ el Galta ${ }^{1,3}$, MF Rudd', EL Webb', H Bridle ${ }^{2}$, T Eisen $^{2,3}$, RS Houlston $^{* 1,3}$ and the GELCAPS \\ Consortium $^{4}$
}

'Section of Cancer Genetics, Institute of Cancer Research, Brookes Lawley Building, Sutton, Surrey SM2 5NG, UK; '2Department of Oncology, University of Cambridge, Cambridge CB2 2RE, UK

\begin{abstract}
Functional nonsynonymous single-nucleotide polymorphisms (nsSNPs) of folate metabolism genes can influence the methylation of tumour suppressor genes, thereby potentially impacting on tumour behaviour. To investigate whether such polymorphisms influence lung cancer survival, we genotyped 14 nsSNPs mapping to methylene-tetrahydrofolate reductase (MTHFR), methionine synthase (MTR), methionine synthase reductase (MTRR); DNA methyltransferase (DNMT2), methylenetetrahydrofolate dehydrogenase (MTHFDI) and methenyltetrahydrofolate synthetase (MTHFS) in 619 Caucasian women with incident disease, 465 with non-small cell (NSCLC) and 154 with small cell lung cancer (SCLC). The most significant association detected was with MTHFS Thr202Ala, with carriers of variant alleles having a worse prognosis (hazard ratio $(H R)=1.49 ; 95 \%$ confidence interval: I. I4- I.94). Associations were also detected between overall survival (OS) in SCLC and homozygosity for MTHFR 222Val (HR = 1.92; I.03-3.58) and between OS from NSCLC and MTRR I 75Leu carrier status ( $\mathrm{HR}=1.36 ; 1.06-1.75)$. While there is evidence that variation in the folate metabolism genes may influence prognosis from lung cancer, current data are insufficiently robust to distinguish individual patient outcome. British Journal of Cancer (2007) 97, 247-252. doi: I0.1038/sj.bjc.6603830 www.bjcancer.com
\end{abstract}

Published online 29 May 2007

(c) 2007 Cancer Research UK

Keywords: lung cancer; SNP; prognosis; folate metabolism

Lung cancer is a major cause of cancer mortality worldwide. In the United Kingdom, it accounts for more than 33000 cancer deaths each year (Cancer Research UK). Despite improvements in treatment in recent years, the prognosis from the disease has only marginally improved with 5-year survival rates for both small (SCLC) and non-small cell lung cancer (NSCLC), typically being no better than 15\% (Jemal et al, 2002). While the major prognostic determinant in lung cancer is stage at presentation, there is variability in survival for patients with same-stage disease. Hence, it is advantageous to identify further prognostic markers, which may aid identification of those patients who will benefit from therapeutic interventions. Furthermore, identifying genes which influence prognosis has the potential to aid the identification of pathways that will be targeted for therapeutic interventions.

Aberrant DNA methylation is recognised as being a common feature of human neoplasia, CpG island hypermethylation and global genomic hypomethylation occurring simultaneously in tumours including lung cancers. Moreover, the cellular profile of DNA hypermethylation has been implicated in progression and metastasis of lung cancer (Nakamura et al, 2003; Shimamoto et al, 2004).

\footnotetext{
* Correspondence: Professor RS Houlston;

E-mail: richard.houlston@icr.ac.uk

${ }^{3}$ These authors contributed equally

${ }^{4}$ List of GELCAPS Consortium collaborators available on request http:// www.cancerresearchuk.org/aboutcancer/statistics Cancer Research UK, 2004, www.ncrn.org.uk/portfolio/data.asp?ID =78I (GELCAPS)

Received 7 March 2007; revised 20 April 2007; accepted 9 May 2007; published online 29 May 2007
}

Variants of folate metabolism pathway (Figure 1) genes such as functional polymorphisms of 5,10-methylene-tetrahydrofolate reductase (MTHFR), affect methylation of DNA and tumour suppressor genes (Kamiya et al, 1998; Paz et al, 2002), thereby potentially impacting on tumour behaviour. This coupled with the observation that polymorphisms of this pathway can affect the efficacy of cytotoxic drugs (Maring et al, 2005) has provided a strong rationale for evaluating such variants as prognostic factors.

Here, we report the impact of polymorphic variation within the folate metabolism pathway genes MTHFR, methionine synthase $(M T R)$, methionine synthase reductase $(M T R R)$, DNA methyltransferase (DNMT2), methylenetetrahydrofolate dehydrogenase (MTHFD1) and methenyltetrahydrofolate synthetase (MTHFS) on lung cancer prognosis in 619 patients. We based our analysis on nonsynonymous single-nucleotide polymorphisms (nsSNPs) as these alter the amino-acid sequence of expressed proteins and are most likely to have functional consequences.

\section{METHODS}

\section{Patients}

Patients with lung cancer were ascertained through the Genetic Lung Cancer Predisposition Study (GELCAPS). Full details about the design and conduct of the study can be obtained elsewhere (Matakidou et al, 2005). Briefly, patients were recruited through oncology centres in the UK specializing in the management of lung cancer. To ensure that data and samples were collected from bona fide lung cancer cases and avoid issues of bias from survivorship, only incident cases with histologically or cytologically (only if not 
adenocarcinoma) confirmed primary disease were ascertained. Demographic characteristics (sex, date of birth, ethnic group, area of residence and country of birth, smoking history, history of lung cancer in a first degree relative), treatment and clinical follow-up were collected from cases using standardized questionnaire and proformas. The current analysis is based on 619 female patients all of whom are white Caucasians. Patient characteristics are detailed in Table 1. Ethical approval for the study was obtained from the London Multi-Centre Research Ethics Committee (MREC/98/2/67) in accordance with the tenets of the Declaration of Helsinki. All participants provided informed consent.

\section{Genotyping}

DNA was extracted from EDTA-venous blood samples using a salt extraction procedure and quantified by Picogreen. The 13 nsSNPs genotyped, DNMT2 Asp112Tyr, MTHFD1 Lys134Arg, MTHFD1 Arg653Gln, MTHFR Ala222Val, MTHFR Arg594Gln, MTHFR Thr202Ala, MTR Asp919Gly, MTRR His595Tyr, MTRR Ser175Leu, MTRR Lys350Arg, MTRR Pro450Arg, MTRR Arg415Cys, MTRR Ser257Thr. These SNPs were chosen on the basis of being polymorphic in Caucasians, feasibility of genotyping and established functional impact of the sequence changes. Genotyping was conducted by means of Illumina Sentrix Bead Arrays (Illumina, San Diego, CA, USA) according to the manufacturer's protocols (details available on request).

\section{Statistical methods}

Associations between survival and demographic and clinical variables were assessed by means of the $\chi^{2}$ and Fisher's exact tests. Testing for population substructure was based on examining the distribution of SNP genotypes for evidence of HardyWeinberg disequilibrium. Overall survival (OS) of patients was the end point of the analyses. Survival time was calculated from the date of diagnosis of lung cancer to the date of death. Patients who were not deceased were censored at the date of last contact. Median follow-up time was computed among censored observations only. Kaplan-Meier survival curves according to genotype were generated and the homogeneity of the survival curves was tested using the log-rank test. Cox regression analysis (Klein and Moeschberger, 1997) was used to estimate hazard ratios (HRs) and their 95\% confidence intervals (CI) while adjusting for radiotherapy and stage. Likelihood ratio testing for the inclusion of covariates and interaction terms was performed to determine the
Table I Patient demographic and follow-up characteristics

\begin{tabular}{|c|c|}
\hline & No. of patients (\%) \\
\hline Total & 619 \\
\hline Mean age (years) & 64.8 \\
\hline \multicolumn{2}{|l|}{ Smoking habits } \\
\hline Non-smokers & $49(8)$ \\
\hline Smokers & $570(92)$ \\
\hline \multicolumn{2}{|l|}{ Histology } \\
\hline $\mathrm{SCLC}$ & $154(25)$ \\
\hline NSCLC & $465(75)$ \\
\hline Squamous & $180(30)$ \\
\hline Adenocarcinoma & $164(27)$ \\
\hline \multicolumn{2}{|l|}{ Tumour stage, by histology } \\
\hline \multicolumn{2}{|l|}{ SCLC } \\
\hline Limited & $66(43)$ \\
\hline Extensive & $86(57)$ \\
\hline \multicolumn{2}{|l|}{ NSCLC } \\
\hline I & $57(13)$ \\
\hline$\|$ & $68(15)$ \\
\hline III & $196(43)$ \\
\hline IV & $130(29)$ \\
\hline Median survival time (months) & 16.2 \\
\hline Events (deaths) & $389(62.8)$ \\
\hline Median survival time, months, by histology and stage & \\
\hline \multicolumn{2}{|l|}{ SCLC } \\
\hline Limited & 17.8 \\
\hline Extensive & 11.1 \\
\hline All stages & 13.5 \\
\hline \multicolumn{2}{|l|}{ NSCLC } \\
\hline I & 49.2 \\
\hline$\|$ & 31.9 \\
\hline III & 16.2 \\
\hline IV & 11.5 \\
\hline All stages & 17.6 \\
\hline
\end{tabular}

$\mathrm{NSCLC}=$ non-small cell lung cancer; $\mathrm{SCLC}=$ small cell lung cancer.

best-fitting model. For each SNP, HRs were generated using common allele homozygotes as the reference group (unless otherwise specified). For polymorphisms with fewer than five 
minor allele homozygotes, minor allele homozygote genotypes were combined with heterozygotes. In addition, to study the impact of individual SNPs on survival, we evaluated OS as a function of the number of 'risk alleles' carried. In this analysis, risk was trichotomised into low, medium and high-risk categories. Owing to the exploratory nature of this study, we reported nominal statistical associations for all analyses. We recognise that examining multiple SNPs risks identification of false associations. However, correction for multiple testing may increase the risk of type II errors (Perneger, 1998). Accordingly, we present uncorrected $P$-values but recognise our exploratory findings require confirmation in another study. This approach minimises loss of true positive results but allows false positive results to be identified (Perneger, 1998; Cuzick, 1999). To adjust for multiple testing, we multiplied $P$-values of each individual test statistic by the number of SNPs in the corresponding gene to obtain a genewide $P$-value, the global $P$-value being the product of the genewide $P$-value and the number of genes. Statistical analyses were undertaken using S-Plus (Version 8, Insightful Corporation, USA). The power to demonstrate a relationship between SNP genotype and OS was estimated using sample size formulae for comparative binomial trials (Farrington and Manning, 1990). In all analyses, a $P$-value of 0.05 was considered statistically significant. To assess the level of linkage disequilibrium (LD) between SNPs, we calculated the pairwise LD measure $r^{2}$ between markers mapping to the same gene using the programme PHASE (Stephens et al, 2001) that implements the Monte Carlo Markov Chain procedure to estimate two-locus haplotype frequencies. This information was used to investigate the relationship between haplotypes and OS.

\section{Bioinformatic analysis}

We applied two in silico algorithms, Polymorphism Phenotyping (PolyPhen) and the Sorting Intolerant from Tolerant to predict the putative impact of missense variants on protein function $(\mathrm{Ng}$ and Henikoff, 2002; Ramensky et al, 2002). Sorting Intolerant from Tolerant and PolyPhen scores were classified according to the established criteria (Ng and Henikoff, 2002; Xi et al, 2004).

\section{RESULTS}

\section{Study population and SNP genotype distributions}

One hundred and fifty-four of the patients (25\%) had SCLC, somewhat less than half $(43 \%)$ presenting with limited disease. Of the 465 patients with NSCLC, 57 (13\%) had stage I, 68 (15\%) had stage II, 196 (43\%) had stage III and 130 (29\%) had stage IV disease at diagnosis. The majority of patients with limited stage SCLC had been treated with a combination of radical radiotherapy and chemotherapy, while all patients received chemotherapy (Table 1). The main treatment modality for SCLC patients with extensive disease was chemotherapy. Patients with early stage NSCLC (stage I and II disease) were mainly treated with surgical resection of the primary tumour, while about one-third received chemotherapy and radical radiotherapy. The mainstay treatment modality of patients with stage III and IV NSCLC was chemotherapy. There were $389(62.8 \%)$ deaths in the cohort. Overall the median survival time (MST) was 16.2 months (range 0.03-60.5 months). There were 13 patients with follow-up time less than 1 month, from whom five died. Patients with SCLC had a MST of 17.8 and 11.1 months, if diagnosed with limited and extensive disease, respectively. For NSCLC, by stage, MST ranged from 11.5 months in stage IV patients to 49.2 months in the stage I group. As these survival rates are not significantly different to those documented in audits of lung cancer prognosis (Cancer Research $\mathrm{UK})$, there is no evidence that 'healthy study participant' selection will have biased our analyses.
Surgery, any chemotherapy and treatment specifically with platinum-based compounds did not satisfy the proportional hazards assumption required for the Cox model. Therefore, we used a stratified Cox model, stratifying on these covariates. Stage at presentation, histology, radiotherapy, smoking, family history of lung cancer and age at diagnosis were initially included as covariates and backward stepwise selection procedure was conducted to cover the most parsimonious model. Stage and age were included as categorical and continuous variables, respectively. Other factors were coded as binary variables. Factors significantly influencing patient prognosis were stage at presentation $\left(P<10^{-4}\right)$, histology $(P=0.026)$ and radiotherapy $(P=0.0042)$. Smoking, family history of lung cancer and age at diagnosis did not impact on survival.

\section{Relationship between SNP genotype and prognosis}

For most SNPs genotyped (92\%), minor allele frequencies (MAF) were $5 \%$ or higher. One SNP was however, observed at comparatively low frequencies (i.e. having MAF $<5 \%$ ). There was no evidence in the data set for population stratification based on testing the distribution of SNP genotypes for Hardy-Weinberg disequilibrium. Thirteen nsSNPs in six genes were assayed. Only SNPs S257T, R415C and P450R, and SNPs H595Y and K175L, all mapping to MTRR, were in strong LD (i.e. $r^{2}=1.0$ and $r^{2}=0.81$, respectively). Hence, the relationship between SNP haplotype and prognosis was restricted to this locus.

There was no correlation between the SNP genotype and pathological parameters, (stage and histology), but in view of the differences in biology of NSCLC and SCLC we also examined for relationships between genotypes and prognosis in the two cell types separately. Table 2 details the relationships between SNP genotype and OS from lung cancer obtained from Cox regression analysis.

Significant associations were identified between polymorphic variation in MTHFS, MTHFR and MTRR. Under the Cox proportional hazards model, the HRs for OS from all lung cancer associated with MTHFS Thr202Ala heterozygosity, homozygosity and carrier status were: 1.53 (95\% CI: $1.17-2.01), 1.04$ (95\% CI: $0.38-2.84)$ and 1.49 (95\% CI: $1.14-1.94)$, respectively. KaplanMeier estimates demonstrated that carriers had a shorter MST than patients with the wild-type genotype (MST of 12.9 and 16.7 months, respectively; $P=0.052$; Figure 2). The HRs for OS from NSCLC associated with MTHFS Thr202Ala heterozygosity, homozygosity and carrier status were 1.4 (95\% CI: 1.02-1.92), 1.45 (95\% CI: $0.44-4.71)$ and 1.4 (95\% CI: $1.06-1.91)$, respectively. The HRs for OS from SCLC associated with MTHFS Thr202Ala carrier status was 1.96 (95\% CI: $1.17-3.30)$. For SCLC there were too few homozygotes, hence, these data were pooled with heterozygotes. Variation in MTHFR defined by Ala222Val appeared to influence OS for SCLC in a recessive fashion with HR associated with heterozygote, homozygote and carrier status being 1.08 (95\% CI: $0.70-1.68$ ), 1.92 (95\% CI: $1.03-3.58$ ) and 1.20 (95\% CI: $0.79-1.82$ ), respectively. For this group, Kaplan-Meier estimates showed that variant homozygotes had a shorter MST than patients with other genotypes (MSTs of 10.3 and 14.3 months, respectively; $P=0.025$, Figure 2). MTHFR Arg594Gln influenced OS with HR associated with carrier status being 0.68 (95\% CI: $0.46-1.00)$, and 0.60 (95\% CI: $0.37-0.95)$ in all lung cancer and NSCLC, respectively. For NSCLC, Kaplan-Meier estimates showed that variant carriers had a longer MST than patients with other genotypes (25.4 and 16.2 months, respectively; $P=0.02$; Figure 1 ). There was evidence that carrier status for MTRR Ser175Leu is associated with a poorer prognosis $(\mathrm{HR}=1.14 ; 95 \% \mathrm{CI}: 0.92-1.41)$ albeit borderline significance in all cancers but significant in NSCLC $(\mathrm{HR}=1.36$; 95\% CI: $1.06-1.75)$. For NSCLC patients, MST in carriers was 15.9 compared to 19.8 months in patients with other genotypes $(P=0.09$, Figure 1$)$. A number of other variants were also 
Table 2 Relationship between overall survival from lung cancer and polymorphisms in folate metabolism genes

\begin{tabular}{|c|c|c|c|c|c|c|c|c|c|c|c|c|c|}
\hline \multirow[b]{3}{*}{$\begin{array}{l}\text { Gene } \\
\text { amino acid }\end{array}$} & \multirow[b]{3}{*}{ Genotype } & \multicolumn{4}{|c|}{ All cancer } & \multicolumn{4}{|c|}{ NSCLC } & \multicolumn{4}{|c|}{ SCLC } \\
\hline & & \multirow[b]{2}{*}{$\begin{array}{c}\text { Dead } \\
(n=375)\end{array}$} & \multirow[b]{2}{*}{$\begin{array}{c}\text { Alive } \\
(n=219)\end{array}$} & \multicolumn{2}{|c|}{ Survival } & \multirow[b]{2}{*}{$\begin{array}{c}\text { Dead } \\
(n=27 I)\end{array}$} & \multirow[b]{2}{*}{$\begin{array}{c}\text { Alive } \\
(n=171)\end{array}$} & \multicolumn{2}{|c|}{ Survival } & \multirow[b]{2}{*}{$\begin{array}{c}\text { Dead } \\
(n=104)\end{array}$} & \multirow[b]{2}{*}{$\begin{array}{c}\text { Alive } \\
(n=48)\end{array}$} & \multicolumn{2}{|c|}{ Survival } \\
\hline & & & & HR & $95 \% \mathrm{Cl}$ & & & HR & $95 \% \mathrm{Cl}$ & & & HR & $95 \% \mathrm{Cl}$ \\
\hline DNMT2 & $+/+$ & 259 & 152 & & - & 188 & 120 & & - & 71 & 32 & & - \\
\hline \multirow{3}{*}{ Asp I I2Tyr } & $+/-$ & 111 & 64 & 0.94 & $0.75-1.19$ & 78 & 49 & 0.95 & $0.72-1.25$ & 33 & 15 & 0.97 & $0.63-1.50$ \\
\hline & $-1-$ & 5 & 3 & 0.75 & $0.31-1.83$ & 5 & 2 & 0.81 & $0.33-1.98$ & 0 & 1 & & - \\
\hline & $+1-$ and $-1-$ & & & 0.93 & $0.74-1.17$ & & & 0.94 & $0.72-1.23$ & & & 0.97 & $0.63-1.50$ \\
\hline MTHFDI & $+1+$ & 257 & 146 & & - & 186 & 114 & & - & 71 & 32 & & - \\
\hline \multirow[t]{2}{*}{ Lys I34Arg } & $+/-$ & 105 & 64 & 0.97 & $0.77-1.22$ & 74 & 51 & 1.01 & $0.76-1.33$ & 31 & 13 & 0.83 & $0.53-1.30$ \\
\hline & $\begin{array}{l}-1- \\
+1-\text { and }-1-\end{array}$ & 13 & 9 & 1.46 & $0.83-2.57$ & 11 & 6 & 1.55 & & 2 & 3 & 1.22 & $0.28-5.23$ \\
\hline MTHFDI & $+1+$ & 94 & 60 & & $\begin{array}{c}0.81-1.26 \\
-\end{array}$ & 67 & 51 & & $\begin{array}{c}0.81-1.38 \\
-\end{array}$ & 27 & 9 & & $\begin{array}{c}0.35-1.32 \\
-\end{array}$ \\
\hline Arg653Gln & $+1-$ & 203 & 113 & 0.88 & $0.68-1.12$ & 153 & 88 & 0.96 & $0.71-1.29$ & 50 & 25 & 0.86 & $0.52-1.40$ \\
\hline & $\begin{array}{l}-1- \\
+1-\text { and }-I-\end{array}$ & 78 & 46 & $\begin{array}{l}0.78 \\
0.85\end{array}$ & $\begin{array}{l}0.58-1.07 \\
0.67-1.08\end{array}$ & 51 & 32 & $\begin{array}{l}0.76 \\
0.9\end{array}$ & $\begin{array}{l}0.52-1.11 \\
0.68-1.20\end{array}$ & 27 & 14 & $\begin{array}{l}1.2 \\
0.96\end{array}$ & $\begin{array}{l}0.69-2.06 \\
0.61-1.52\end{array}$ \\
\hline MTHFR & $+1+$ & 153 & 100 & & - & 115 & 78 & & - & 38 & 22 & & - \\
\hline Ala222Val & $+1-$ & 181 & 100 & 1.09 & $0.88-1.36$ & 130 & 77 & 1.09 & $0.84-1.41$ & 51 & 23 & 1.08 & $0.70-1.68$ \\
\hline & $-1-$ & 41 & 19 & 1.37 & $0.96-1.95$ & 26 & 16 & 1.2 & $0.77-1.86$ & 15 & 3 & 1.92 & $1.03-3.58$ \\
\hline & $+1-$ and $-1-$ & & & 1.13 & $0.91-1.40$ & & & 1.1 & $0.86-1.42$ & & & 1.2 & $0.79-1.82$ \\
\hline MTHFR & $+/+$ & 347 & 192 & & - & 252 & 148 & & - & 95 & 44 & & - \\
\hline Arg594Gln & $+/-$ & 28 & 26 & 0.68 & $0.46-1.00$ & 19 & 22 & 0.6 & $0.37-0.95$ & 9 & 4 & 0.95 & $0.48-1.89$ \\
\hline & $-1-$ & 0 & I & & - & 0 & I & & - & 0 & 0 & & - \\
\hline & $+1-$ and $-1-$ & & & 0.68 & $0.46-1.00$ & & & 0.6 & $0.37-0.95$ & & & 0.95 & $0.48-1.89$ \\
\hline MTHFS & $+/+$ & 305 & 182 & & - & 220 & 142 & & - & 85 & 40 & & - \\
\hline Thr202Ala & $+/-$ & 66 & 33 & 1.53 & $1.17-2.01$ & 48 & 26 & 1.4 & $1.02-1.92$ & 18 & 7 & 1.96 & $1.17-3.30$ \\
\hline & $-1-$ & 4 & 4 & 1.04 & $0.38-2.84$ & 3 & 3 & 1.45 & $0.44-4.71$ & 1 & 1 & & - \\
\hline & $+1-$ and $-1-$ & & & 1.49 & $1.14-1.94$ & & & 1.4 & $1.02-1.91$ & & & 1.96 & $1.17-3.30$ \\
\hline MTR & $+/+$ & 248 & 138 & & - & 183 & 104 & & - & 65 & 34 & & - \\
\hline Asp919Gly & $+1-$ & 110 & 70 & 0.94 & $0.75-1.19$ & 76 & 59 & 0.88 & $0.67-1.15$ & 34 & 11 & 1.1 & $0.72-1.69$ \\
\hline & $-1-$ & 17 & 11 & 1.44 & $0.88-2.36$ & 12 & 8 & 1.35 & $0.75-2.45$ & 5 & 3 & 1.65 & $0.65-4.15$ \\
\hline & $+1-$ and $-1-$ & & & 0.99 & $0.80-1.23$ & & & 0.92 & $0.71-1.19$ & & & 1.15 & $0.77-1.73$ \\
\hline MTRR & $+/+$ & 302 & 172 & & - & 219 & 133 & & - & 83 & 39 & & - \\
\hline His595Tyr & $+1-$ & 70 & 45 & 1.03 & $0.79-1.34$ & 51 & 36 & 0.99 & $0.73-1.35$ & 19 & 9 & 1.11 & $0.67-1.82$ \\
\hline & $-1-$ & 3 & 2 & 1.02 & $0.32-3.25$ & 1 & 2 & & - & 2 & 0 & & - \\
\hline & $+1-$ and $-1-$ & & & 1.03 & $0.79-1.34$ & & & 0.99 & $0.73-1.35$ & & & 1.11 & $0.67-1.82$ \\
\hline MTRR & $+1+$ & 144 & 92 & & - & 103 & 77 & & - & 41 & 15 & & - \\
\hline Ser 175 Leu & $+1-$ & 177 & 94 & 1.18 & $0.94-1.49$ & 125 & 71 & 1.42 & $1.09-1.87$ & 52 & 23 & 0.81 & $0.52-1.26$ \\
\hline & $-1-$ & 54 & 33 & 1.01 & $0.74-1.39$ & 43 & 23 & 1.21 & $0.85-1.74$ & 11 & 10 & 0.62 & $0.31-1.23$ \\
\hline & $+1-$ and $-1-$ & & & 1.14 & $0.92-1.41$ & & & 1.36 & $1.06-1.75$ & & & 0.77 & $0.50-1.17$ \\
\hline MTRR & $+1+$ & 286 & 164 & & - & 205 & 126 & & - & 81 & 38 & & - \\
\hline Lys350Arg & $+1-$ & 86 & 51 & 1.09 & $0.85-1.39$ & 65 & 42 & 1.09 & $0.82-1.45$ & 21 & 9 & 0.91 & $0.56-1.49$ \\
\hline & $-1-$ & 3 & 4 & 0.79 & $0.25-2.51$ & 1 & 3 & & - & 2 & 1 & & - \\
\hline & $+1-$ and $-1-$ & & & 1.07 & $0.84-1.37$ & & & 1.09 & $0.82-1.45$ & & & 0.91 & $0.56-1.49$ \\
\hline MTRR & $+/+$ & 356 & 210 & & - & 259 & 164 & & - & 97 & 46 & & - \\
\hline Pro450Arg & $+1-$ & 19 & 9 & 1.38 & $0.87-2.20$ & 12 & 7 & 1.41 & $0.78-2.53$ & 7 & 2 & 1.32 & $0.60-2.88$ \\
\hline & $-1-$ & 0 & 0 & & - & 0 & 0 & & - & 0 & 0 & & - \\
\hline & $+1-$ and $-1-$ & & & 1.38 & $0.87-2.20$ & & & 1.41 & $0.78-2.53$ & & & 1.32 & $0.60-2.88$ \\
\hline MTRR & $+/+$ & 356 & 210 & & - & 259 & 164 & & - & 97 & 46 & & - \\
\hline Arg4I5Cys & $+1-$ & 19 & 9 & 1.38 & $0.87-2.20$ & 12 & 7 & 1.41 & $0.78-2.53$ & 7 & 2 & 1.32 & $0.60-2.88$ \\
\hline & $-1-$ & 0 & 0 & & - & 0 & 0 & & & 0 & 0 & & \\
\hline & $+1-$ and $-1-$ & & & 1.38 & $0.87-2.20$ & & & 1.41 & $0.78-2.53$ & & & 1.32 & $0.60-2.88$ \\
\hline MTRR & $+/+$ & 356 & 210 & & - & 259 & 164 & & - & 97 & 46 & & - \\
\hline Ser257Thr & $+1-$ & 19 & 9 & 1.38 & $0.87-2.20$ & 12 & 7 & 1.41 & $0.78-2.53$ & 7 & 2 & 1.32 & $0.60-2.88$ \\
\hline & $\begin{array}{l}-1- \\
+1-\text { and }-1-\end{array}$ & 0 & 0 & & - & 0 & 0 & & $078-253$ & 0 & 0 & & $060-288$ \\
\hline & $+1-$ and $-1-$ & & & 1.38 & $0.87-2.20$ & & & 1.41 & $0.78-2.53$ & & & 1.32 & $0.60-2.88$ \\
\hline
\end{tabular}



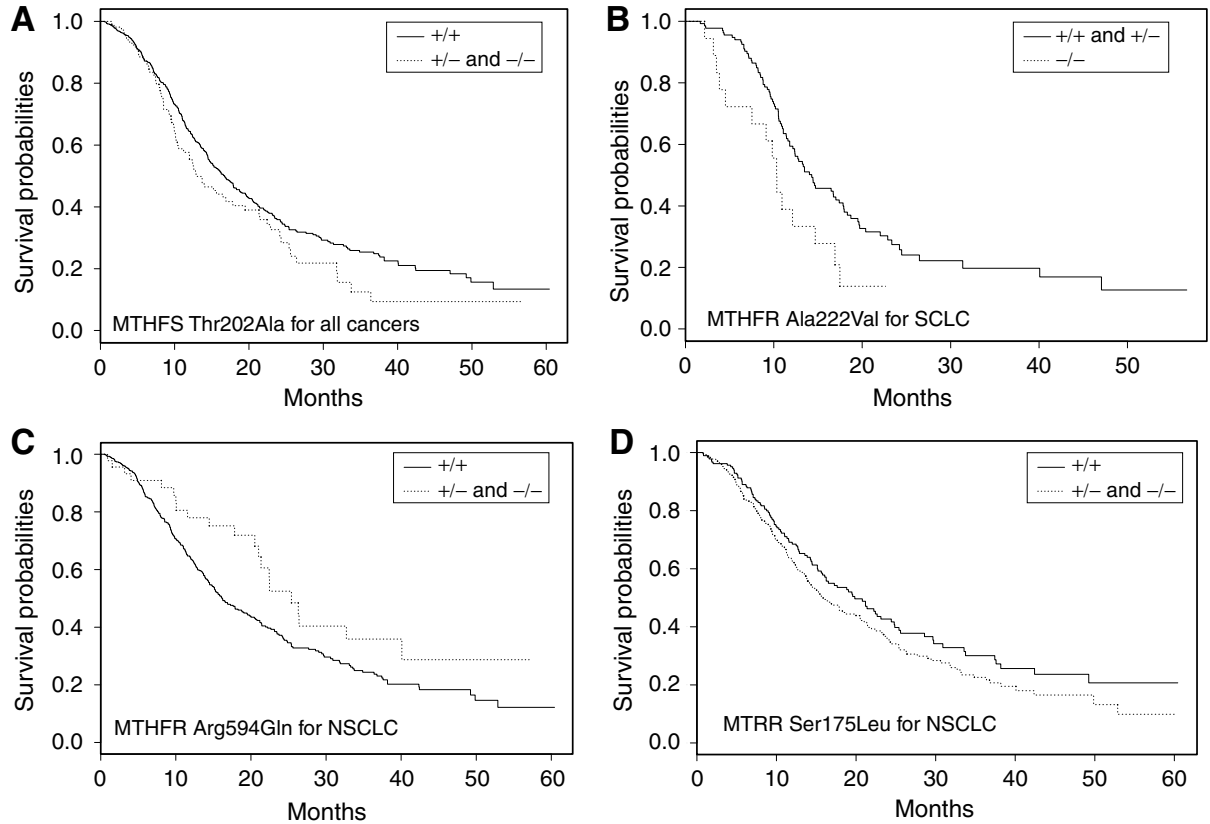

Figure 2 Kaplan-Meier curves for lung cancer patients. (A) Relationship between MTHFS Thr202Ala genotype and prognosis from all lung cancer; (B) relationship between MTHFR Ala222Val genotype and prognosis from SCLC; (C) relationship between MTHFR Ala222Val genotype and prognosis from NSCLC; (D) relationship between MTRR Ser I 75Leu genotype and prognosis from NSCLC. The solid line depicts the survival curve for the reference group. $+I+,+I-$ and $-I-$ refer to the common homozygotes, heterozygotes and rare homozygotes, respectively.

associated with OS (Table 2), but none were individually significant.

Evaluating OS as a function of the number of 'risk alleles' provided no evidence of an interaction between SNPs (data not shown). Finally, we examined for potential interactive effects between SNPs, response to platinum-based chemotherapy and prognosis. None showed nominally significant interactions at the $5 \%$ level.

\section{DISCUSSION}

Major strengths of our study are its large size, the fact that it is population-based, included only patients with incident disease, and has involved the systematic follow-up of patients. We are mindful that it is desirable that studies aimed at identifying prognostic markers should be conducted within the context of a clinical trial to minimise bias. Although bias from non-uniform treatment is a potential confounder in studies of some solid tumours, the management of lung cancer is relatively uniform in the UK, as there are only a restricted number of effective chemotherapeutic agents and prognosis is uniformly poor. Support for this assertion is provided by the fact that survival rates observed in our patient cohort were not different to those expected. It is therefore unlikely that any spurious influences as a consequence of study design will have impacted significantly on our findings. It is well known that the allele frequencies of many SNPs vary among different populations. As our analysis was restricted to white patients, our study findings are unlikely to be confounded by population stratification. The main limitation of our study is the ability to pursue an in-depth examination of the effect of non-genetic factors such as circulating folate levels, which may interact with genotype in defining the clinical behaviour of tumours.

Despite such limitations in this study, we have observed significant evidence for associations between survival and variation in MTHFR, MTHFS and MTRR. Our observation that polymorphic variation in the folate metabolism genes influences cancer prognosis is not without precedent (Alberola et al, 2004). We fully acknowledge that we have not captured all variation defined by nsSNPs mapping to all of the folate metabolism genes but our selection was restricted to validated SNPs that could be robustly genotyped using the analytical platform we employed. For example, it would have been desirable to have genotyped nsSNPs mapping to DNMT1 and DNMT3b, given previously published data implicating variation in these genes in development and prognosis of lung cancer (Kassis et al, 2006; Kim et al, 2006; Wang et al, 2006). However, to date only two common (MAF >0.05) validated nsSNPs map to DNMT1 (Ile311Val and His97Arg) and both unfortunately had low designability for the genotyping platform we employed, thereby precluding evaluation.

We evaluated nsSNPs on the basis that each has the capacity to directly affect the function of expressed proteins, implying a higher probability of being directly causally related to susceptibility. There is good evidence that MTHFR Ala222Val directly affect the function of the expressed protein. For SNPs such as MTHFS Thr202Ala and MTRR Ser175Leu, substitutions are not predicted to be benign. Although such in silico predictions about the functional consequences of amino-acid changes are not definitive, these algorithms have been demonstrated in benchmarking studies to successfully categorise $80 \%$ of amino-acid substitutions (Savas et al, 2004; Xi et al, 2004).

The nature of our study precluded us from formally evaluating SNPs in relation to response to radiotherapy as this was only administered to a small number of patients. Similarly only a small number of patients did not receive platinum-based chemotherapy limiting our ability to robustly detect interactions between this type of therapy, genotype and prognosis. Although there may be differences between NSCLC and SCLC, which may reflect differences in biology of the tumour types, our data did not provide real evidence that folate metabolism variation plays a major role in defining differences in prognosis between these tumour types.

In studies of the type we have conducted, there is the issue of adjustment for multiple comparisons. We assessed 13 polymorphisms in seven genes but because more than one polymorphism 
was tested in some genes, the results are not independent. Hence, for MTHFS Thr202Ala, the statistical threshold for global significance is 0.007 .

Issues of power are also relevant to the formulation of studies seeking to identify polymorphic variants influencing cancer prognosis. The magnitude of any difference in prognosis associated with individual SNPs is likely to be at best modest hence stipulating significance levels of $\sim 10^{-4}$ or less to adjust for multiple testing is inherently unrealistic. For example, for an analysis to have $80 \%$ power to demonstrate a $5 \%$ difference in survival, which is clinically relevant, would require at least 4800 patient samples to be analysed even if the frequency of the at-risk genotype is $50 \%$ stipulating such significance levels. For less frequent genotypes, samples sizes would be impossibly large. On this basis the imposition of very stringent $P$-values (as advocated in genome-wide case-control studies) to outcome studies is questionable creating the serious issue of generating a raft of type II errors (Perneger, 1998).

Despite the strong biologic plausibility and consistency with literature for several individual associations as discussed herein, some of these associations may be false positives as a result of the inherent pitfalls of the candidate gene approach. Hence, individual associations reported in this article must inevitably be interpreted with caution. Nevertheless, even for those true associations, it is unlikely that any individual SNP would have sufficient power to predict clinical outcomes in a disease as complex as cancer. In this context, combined analyses of two or more SNPs in the same pathway are likely to have superior potential to assist in distinguishing different outcome patterns among patients with the same stage disease as even $5-10 \%$ differences in prognosis are relevant in a disease. Furthermore, it is plausible that the impact of variation in the folate metabolism genes is likely to be best seen in situations where the pathway plays a major role in defining the efficacy of chemotherapeutic agents in cancers amenable to treatment with agents such as pyrimidine-antagonists (Maring et al, 2005).

In conclusion, however attractive the notion that polymorphisms of the folate metabolism pathway genes are in defining cancer prognosis, their role in lung cancer on the basis of our data is minor at best and they are unlikely to have clinical utility.

\section{ACKNOWLEDGEMENTS}

We are grateful to patients for their participation. Work was undertaken with support from Cancer Research UK, NCRN, HEAL and Sanofi-Aventis. Athena Matakidou was the recipient of a clinical research fellowship from the Allan J Lerner Fund. We are indebted to Richard Coleman, Christina Fleischmann, Nick Hearle, Rosalind Mutch, Mobshra Qureshi, Elaine Ryder-Mills, Hayley Spendlove and Remben Talaban for sample ascertainment and preparation.

\section{Web Site References}

http://www.cancerresearchuk.org/aboutcancer/statistics Cancer Research UK, 2004

www.ncrn.org.uk/portfolio/data.asp?ID = 781 (GELCAPS)

\section{REFERENCES}

Alberola V, Sarries C, Rosell R, Taron M, de las Penas R, Camps C, Massuti B, Insa A, Garcia-Gomez R, Isla D, Artal A, Munoz MA, Cobo M, Bover I, Gonzalez-Larria JL, Terrasa J, Almenar D, Barcelo R, Diz P, SanchezRonco M, Sanchez JJ (2004) Effect of the methylenetetrahydrofolate reductase $\mathrm{C677 \textrm {T }}$ polymorphism on patients with cisplatin/gemcitabinetreated stage IV non-small-cell lung cancer. Clin Lung Cancer 5: 360 - 365

Cuzick J (1999) Interaction, Subgroup Analysis and Sample Size Metabolic polymorphisms and cancer Lyon: IARC Scientific Publications

Farrington CP, Manning G (1990) Test statistics and sample size formulae for comparative binomial trials with null hypothesis of non-zero risk difference or non-unity relative risk. Stat Med 9: 1447-1454

Jemal A, Thomas A, Murray T, Thun M (2002) Cancer statistics, 2002. CA Cancer J Clin 52: $23-47$

Kamiya H, Kawakami K, Miyanaga T, Omura K, Oda M, Murakami S, Watanabe Y (1998) A methylenetetrahydrofolate reductase polymorphism is associated with expression of p16 in human lung cancer. Oncol Rep 5: $911-914$

Kassis ES, Zhao M, Hong JA, Chen GA, Nguyen DM, Schrump DS (2006) Depletion of DNA methyltransferase 1 and/or DNA methyltransferase $3 b$ mediates growth arrest and apoptosis in lung and esophageal cancer and malignant pleural mesothelioma cells. J Thorac Cardiovasc Surg 131: $298-306$

Kim H, Kwon YM, Kim JS, Han J, Shim YM, Park J, Kim DH (2006) Elevated mRNA levels of DNA methyltransferase-1 as an independent prognostic factor in primary nonsmall cell lung cancer. Cancer 107: $1042-1049$

Klein J, Moeschberger M (1997) Survival Analysis: Techniques for Censored and Truncated Data Statistics for biology and health Berlin: Springer

Maring JG, Groen HJ, Wachters FM, Uges DR, de Vries EG (2005) Genetic factors influencing pyrimidine-antagonist chemotherapy. Pharmacogenomics J 5: $226-243$

Matakidou A, Eisen T, Bridle H, O’Brien M, Mutch R, Houlston RS (2005) Case-control study of familial lung cancer risks in UK women. Int $J$ Cancer 116: $445-450$

Nakamura H, Saji H, Idiris A, Kawasaki N, Hosaka M, Ogata A, Saijo T, Kato H (2003) Chromosomal instability detected by fluorescence in situ hybridization in surgical specimens of non-small cell lung cancer is associated with poor survival. Clin Cancer Res 9: $2294-2299$

Ng PC, Henikoff S (2002) Accounting for human polymorphisms predicted to affect protein function. Genome Res 12: $436-446$

Paz MF, Avila S, Fraga MF, Pollan M, Capella G, Peinado MA, SanchezCespedes M, Herman JG, Esteller M (2002) Germ-line variants in methyl-group metabolism genes and susceptibility to DNA methylation in normal tissues and human primary tumors. Cancer Res 62: 4519-4524

Perneger TV (1998) What's wrong with Bonferroni adjustments. BMJ 316: $1236-1238$

Ramensky V, Bork P, Sunyaev S (2002) Human non-synonymous SNPs: server and survey. Nucleic Acids Res 30: 3894-3900

Savas S, Kim DY, Ahmad MF, Shariff M, Ozcelik H (2004) Identifying functional genetic variants in DNA repair pathway using protein conservation analysis. Cancer Epidemiol Biomarkers Prev 13: $801-807$

Shimamoto T, Ohyashiki JH, Hirano T, Kato H, Ohyashiki K (2004) Hypermethylation of E-cadherin gene is frequent and independent of p16INK4A methylation in non-small cell lung cancer: potential prognostic implication. Oncol Rep 12: 389-395

Stephens M, Smith NJ, Donnelly P (2001) A new statistical method for haplotype reconstruction from population data. Am J Hum Genet 68: 978-989

Wang J, Walsh G, Liu DD, Lee JJ, Mao L (2006) Expression of \{Delta\}DNMT3B variants and its association with promoter methylation of p16 and RASSF1A in primary non-small cell lung cancer. Cancer Res 66: $8361-8366$

Xi T, Jones IM, Mohrenweiser HW (2004) Many amino acid substitution variants identified in DNA repair genes during human population screenings are predicted to impact protein function. Genomics 83: $970-979$ 